Supporting Information

\title{
Interface of Electrogenic Bacteria and Reduced \\ Graphene Oxide: Energetics and Electron Transport
}

\author{
Sheldon Cotts $¥$, Bijentimala Keisham ${ }^{\ddagger}$, Jay Rawal and Vikas Berry* \\ Department of Chemical Engineering, University of Illinois at Chicago \\ *vikasb@uic.edu
}

†S.C. and B.K. are co-first authors. These authors contributed equally to this work. 


\section{S1. Experimental Methods}

rGO synthesis

Graphene oxide (GO) was synthesized by a modified Hummers method. Here, 1.0 gram

of 7 mesh graphite flakes (Sigma-Aldrich) was added to a beaker in an ice bath. $40 \mathrm{ml}$ of

Sulfuric acid (Fisher Chemical, 96\%) and $6.6 \mathrm{ml}$ of Nitric acid (Sigma-Aldrich, 70\%) were

added to the beaker and stirred for 40 min. 5 grams of potassium permanganate (Sigma-

Aldrich, $\geq 99.0 \%$ ) was added slowly to keep the reaction temperature below $20{ }^{\circ} \mathrm{C}$ during

addition. The reaction solution was stirred for $30 \mathrm{~min}$ at $40^{\circ} \mathrm{C}$. $30 \mathrm{ml}$ of $20 \%$ Hydrogen

peroxide (Fisher Chemical, 50\%) was carefully added to the solution maintaining a

temperature of $<60^{\circ} \mathrm{C}$ to quench the reaction. The solution was diluted with $200 \mathrm{ml}$ of

deionized water and later vacuum filtered to recover a vibrant yellow graphene oxide

solution. Further, the GO solution was dialyzed for a week in a $2 \mathrm{k}$ MWCO cellulose bag

(Thermo Fisher, slide-a-lyzer flask) to remove the residual ions. Finally, reduced

graphene oxide ( $\mathrm{rGO}$ ) was produced by hydrothermal reduction at $180^{\circ} \mathrm{C}$ for 4 hours.

Geobacter Sulfurreducens media preparation and inoculation 
Geobacter medium was prepared by adding $1.5 \mathrm{~g}$ of Ammonium chloride (Fisher Chemical, $+99 \%$ ), $0.6 \mathrm{~g}$ of Sodium phosphate monobasic (Sigma-Aldrich, $\geq 99.0 \%$ ), 0.1

$\mathrm{g}$ of Potassium chloride (Sigma-Aldrich, $\geq 99.0 \%$ ), $2.5 \mathrm{~g}$ of Sodium bicarbonate (Mallinckrodt), $0.82 \mathrm{~g}$ of Sodium acetate (Sigma-Aldrich, $\geq 99.0 \%$ ), $10 \mathrm{ml}$ of Wolfe's Vitamin Solution (ATCC ${ }^{\circledR}$ MD-VS ${ }^{\text {TM}}$ ) and $10.0 \mathrm{ml}$ of modified Wolfe's Minerals (ATCC ${ }^{\circledR}$ MD-TMS ${ }^{\mathrm{TM}}$ ) to $1.0 \mathrm{~L}$ of distilled water. The medium was autoclaved at $121^{\circ} \mathrm{C}$ for 15 mins. $8.0 \mathrm{~g}$ of filter-sterilized Sodium fumarate (Sigma-Aldrich, $\geq 99.0 \%$ ) was then added to the sterilized medium to reduce it. Under anaerobic conditions $\left(80 \% \mathrm{~N}_{2}\right.$ and $\left.20 \% \mathrm{CO}_{2}\right)$, the Geobacter sulfurreducens $\left(\right.$ ATCC $^{\circledR} 51573^{\text {TM }}$ ) vial was thawed and inoculated in the prepared medium. The bacterial solution was subsequently incubated at $26^{\circ} \mathrm{C}-30^{\circ} \mathrm{C}$ for 2 weeks.

Device construction

The device consists of an anode and cathode chamber separated by a proton exchange membrane (Fuel Cell Store, Nafion 211). The anode and cathode are both $8.75 \mathrm{~cm}^{2}$ pieces of carbon felt (Fuel Cell Store, AvCarb G200). The anode is connected to a 3.30 $\mathrm{k} \Omega$ resistor that connects to a preamplifier (Stanford Research Systems, SRS570) that 
ends at the multimeter (Keithley 2110/2612) data acquisition system. The anode compartment is inoculated with geobacter suspension and an organic substrate of 0.01 M Sodium Acetate (Sigma Aldrich, $\geq 99 \%$ ). The cathode compartment contains a solution of $0.02 \mathrm{M}$ potassium hexacyanoferrate (Sigma Aldrich, $\sim 99 \%$ ). Once the electrical generation stabilizes, the rGO solution is distributed to the anode via a syringe.

\section{Raman analysis}

Graphene (rGO) and geobacter system was probed using WITEC Alpha 300-RA Raman spectrometer with a laser excitation wavelength of $532 \mathrm{~nm}$. All the samples were studied using a $100 \mathrm{X}$ objective with an exposure time of $\sim 5$ mins. The Raman spot size is $\sim 700 \mathrm{~nm}$ as determined using the equation:

$$
\text { Spot size }=\frac{1.22 \lambda}{N A}
$$

Where $\lambda$ is the laser wavelength and NA is the numerical aperture ( 0.9 for $100 \mathrm{X}$ objective).

SEM analysis 
Field Emission SEM, the JSM-6320F, was used to obtain high-resolution micrographs of rGO - bacterial interface. A low accelerating voltage of $2 \mathrm{kV}$ with a working distance of $2.0 \mathrm{~mm}$ was chosen to optimize the image.

\section{S2. Control Experiment}

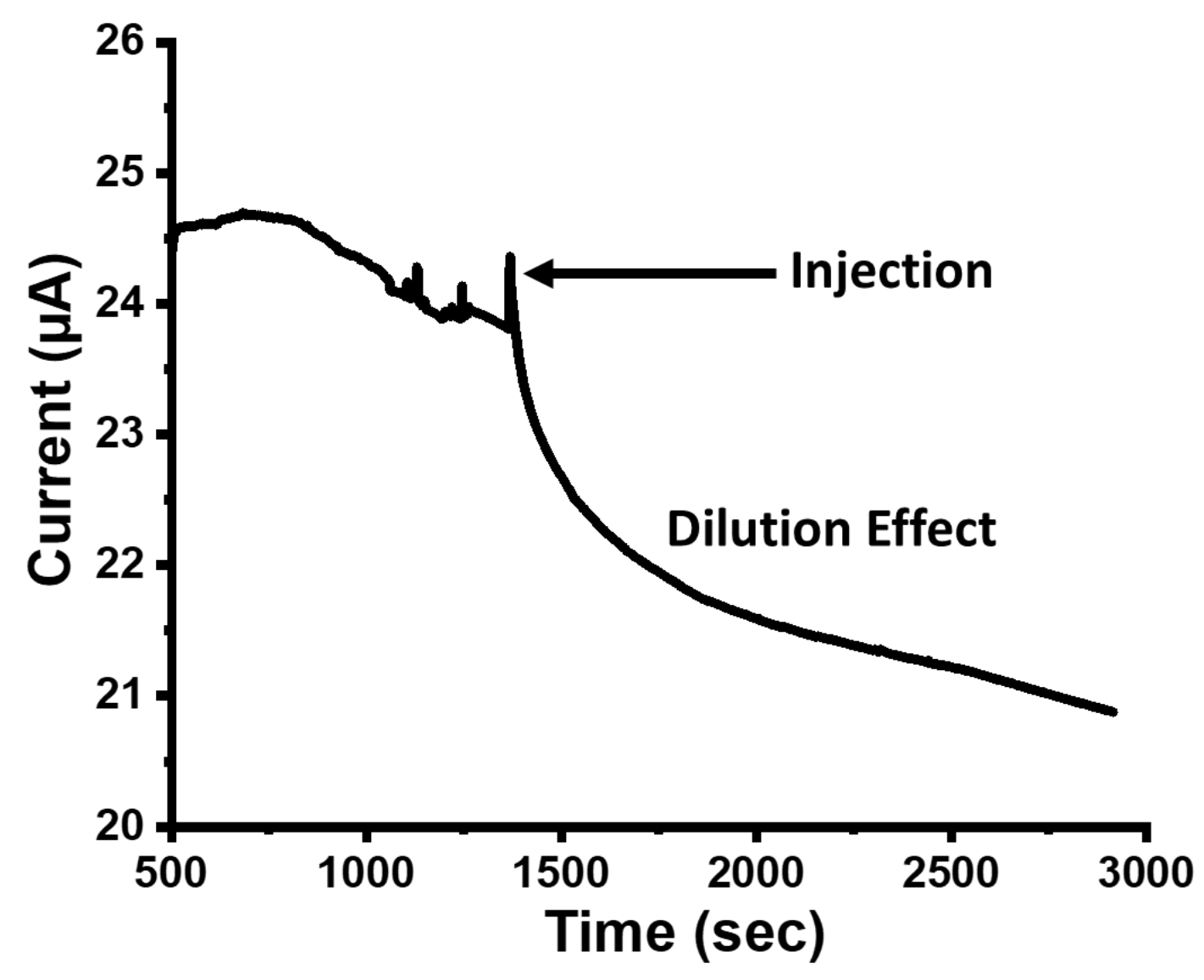

Figure S1. Current response for the MFC construct during operation. DI water was injected at the indicated point as a control experiment to observe the effect on the device from the injection. An instant increase is observed due to the convection on the electrode surface followed by a steady decline in current due to the dilution of the substrate inside of the anodic chamber. The dilution is proportional to the current by decreasing the biocatalyst's access to the substrate, an operational current drop is observed. 


\section{S3. Concentration effect on device current response}

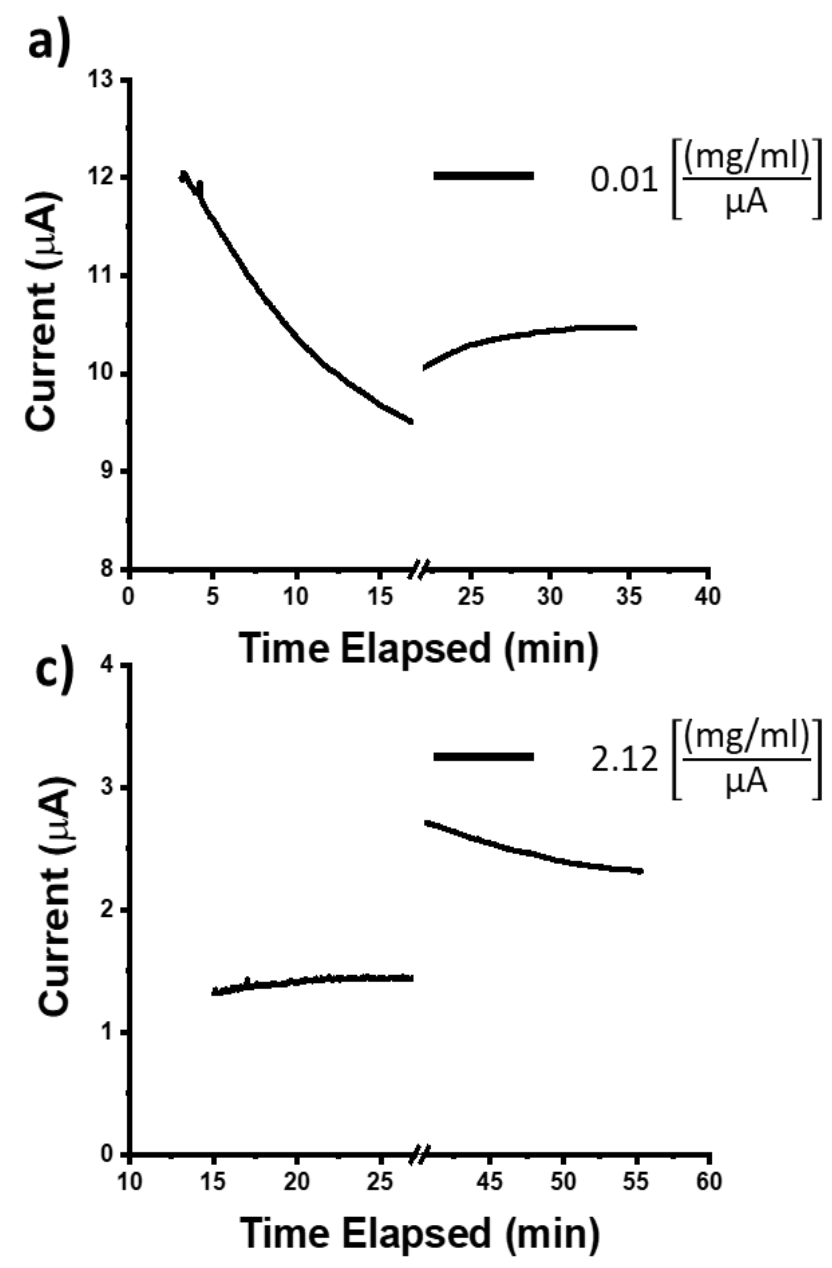

b)
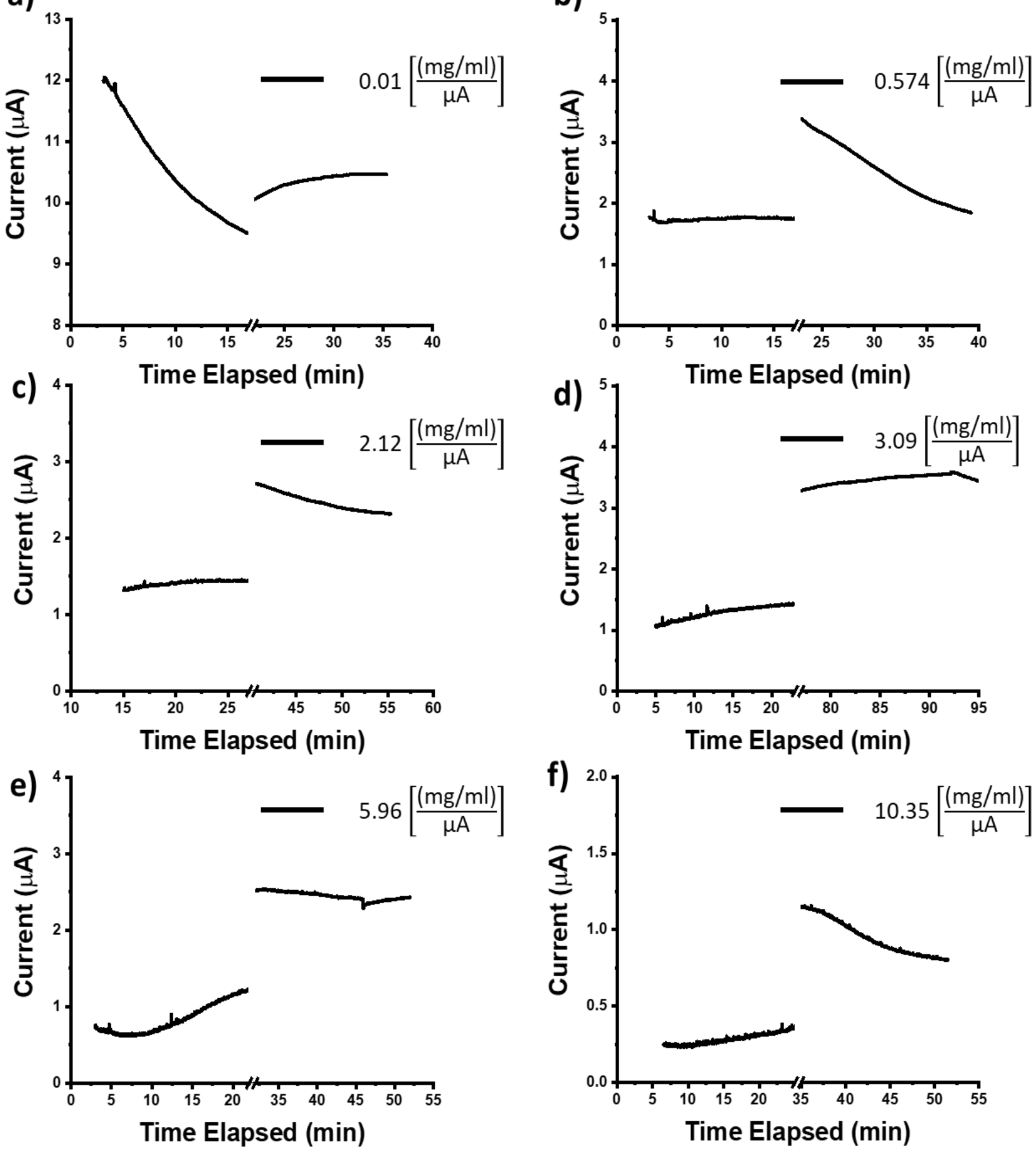

Figure S2. Raw current data collected by varying rGO (reduced graphene oxide) concentration inside device, as shown in figures a) - f). The rGO solution was injected in the anodic chamber and allowed to operate until completion. The data has been truncated to show the current output of the device before and after the injection. The convection effect has not been illustrated and it was not involved in the calculations. The variation in the current is directly related to the amount of biocatalyst inside the apparatus and the cell count was not controlled. 


\section{S4. Raman characterization}

\section{G peak intensity $\longrightarrow$}

a)

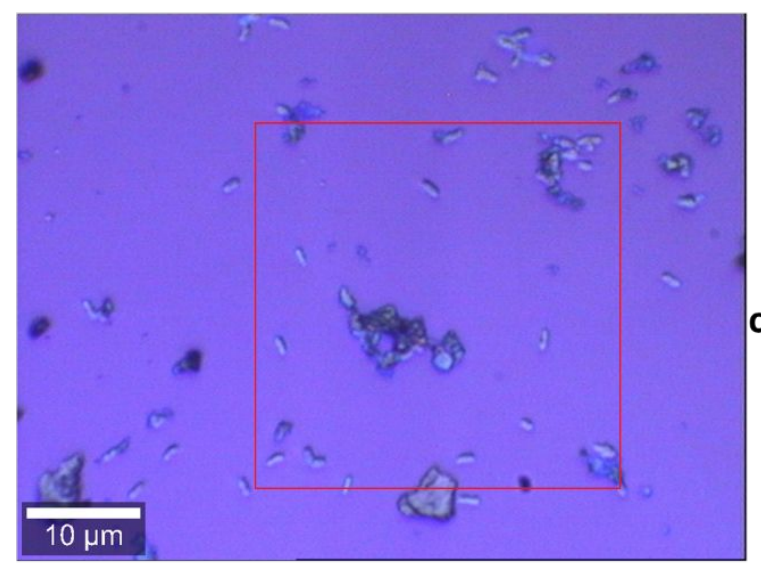

G peak position

b)

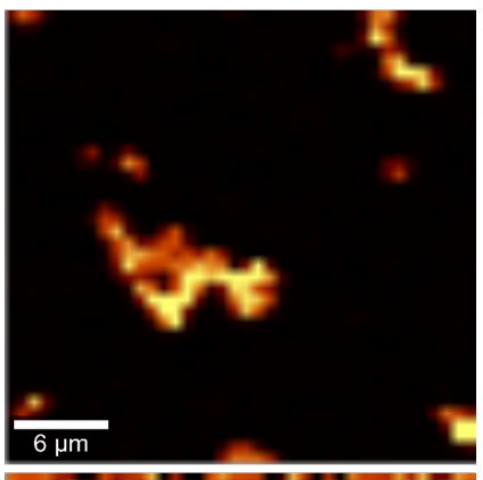

4312 CCD cts

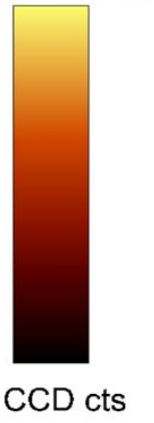

c)

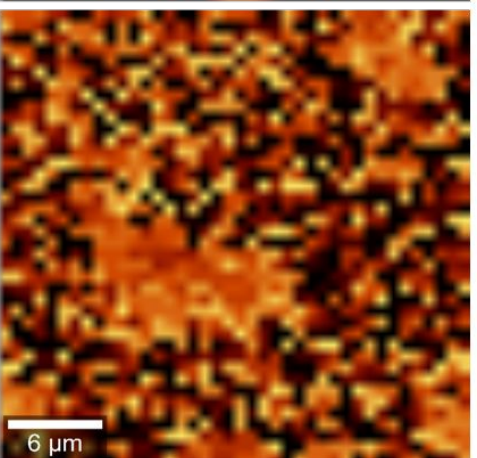

$1624 \mathrm{rel} .1 / \mathrm{cm}$

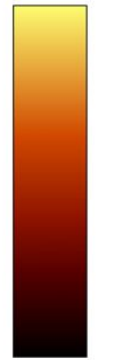

$1550 \mathrm{rel} .1 / \mathrm{cm}$

Figure S3. Raman characterization of rGO-geobacter interface. a) Optical image showing rGO and Geobacter on $\mathrm{SiO}_{2} / \mathrm{Si}$ substrate, scale $10 \mu \mathrm{m}$. The red square represents the scanned area. b) and c) shows the $\mathrm{G}$ peak intensity and position, respectively, of the interfaced rGO

\section{S5. Cell viability assay}
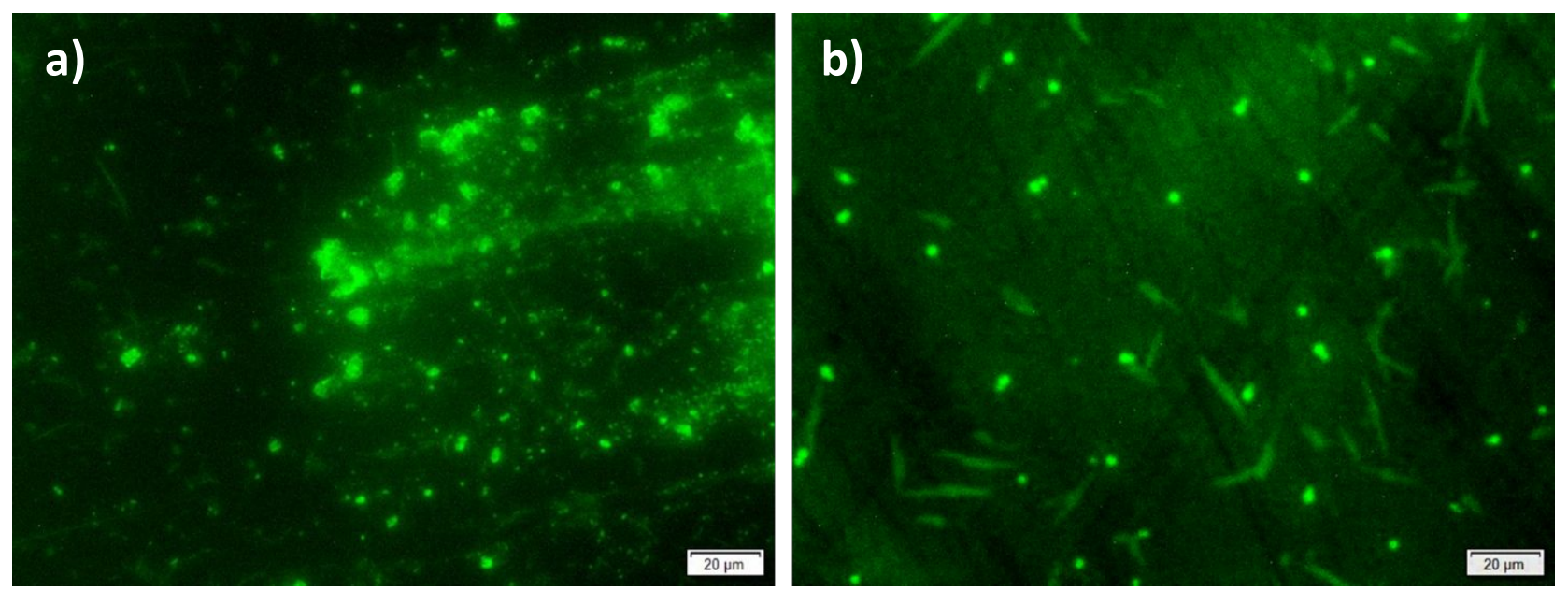

Figure S4. Live-dead bacterial assay. Geobacter cell solution was incubated with rGO solution overnight to perform the live-dead assay. Significant bacterial cells were observed to be still alive as shown in the images a) and b). 


\section{S6. Summarized results}

Table S1. Summary of the effect of different concentrations of rGO associated with the performance of the MFC device.

\begin{tabular}{|l|l|l|l|}
\hline Devices & $\begin{array}{l}\text { Normalized rGO } \\
\text { Concentration } \\
{[(\mathrm{mg} / \mathrm{ml}) / \mu \mathrm{A}]}\end{array}$ & $\begin{array}{l}\text { Average Electron } \\
\text { Transfer Rate }\left(10^{6}\right)\end{array}$ & Device Power density \\
\hline 1 & $0(\mathrm{DI}$ water $)$ & 0.721 & $-28.0 \%$ \\
\hline 2 & 0.01 & 0.989 & $-1.07 \%$ \\
\hline 3 & 0.6 & 1.33 & $33.2 \%$ \\
\hline 4 & 2.12 & 2.07 & $107 \%$ \\
\hline 5 & 3.09 & 2.67 & $168 \%$ \\
\hline 6 & 5.96 & 2.91 & $239 \%$ \\
\hline 7 & 10.35 & 3.42 & \\
\hline
\end{tabular}




\section{S7. XPS characterization}
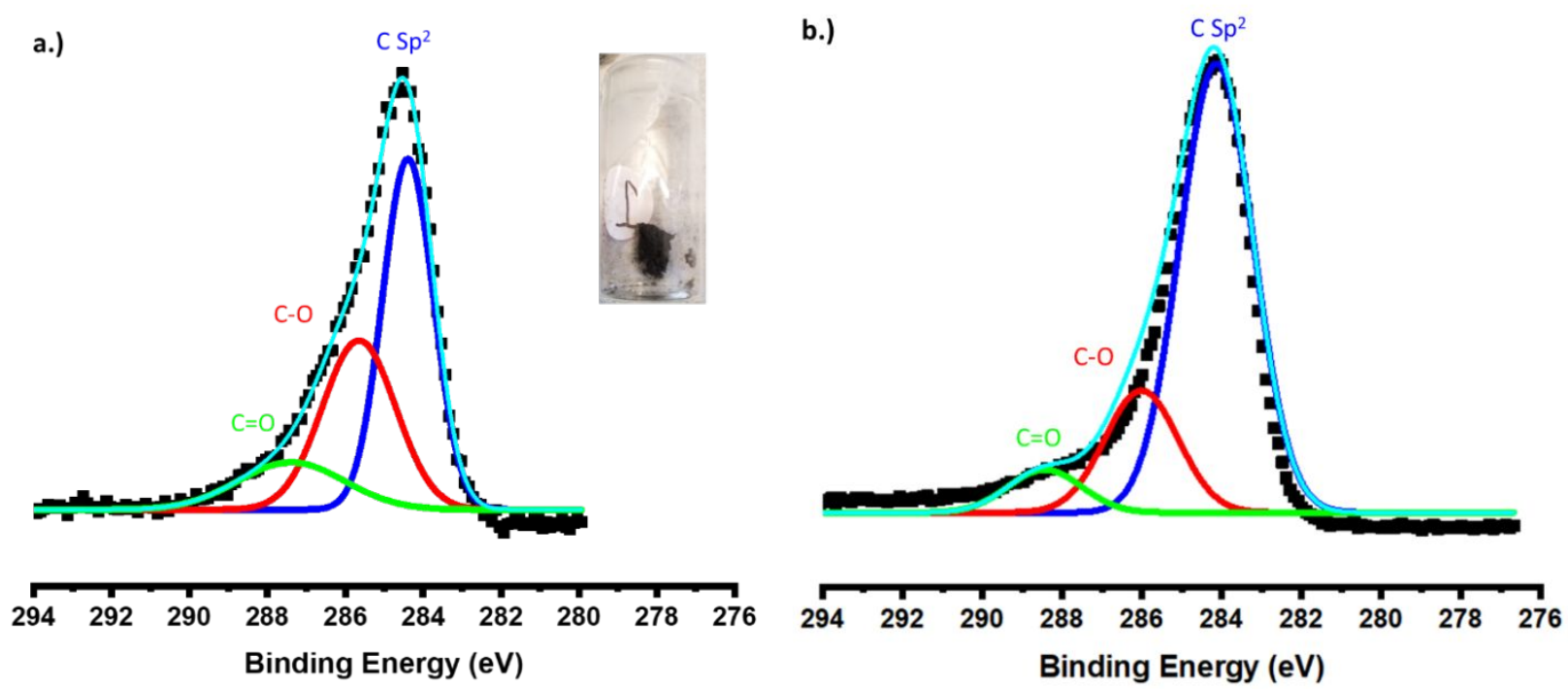

Figure S5. XPS spectra of the C1S peak of both (a) $\mathrm{rGO}$ and (b) CVD graphene on $\mathrm{SiO}_{2}$ substrate. (Insert) Picture of rGO powder after lyophilization.

The XPS analysis shows that the $\mathrm{sp}^{2}$ hybridized carbon and functionalization of different carbonoxygen bonding are observed for both rGO and CVD graphene samples (Figure S7). The CVD graphene has intensity ratios of 3.68 for $\frac{I_{s p^{2}}}{I_{c-o}}$ and 10.46 for $\frac{I_{s p^{2}}}{I_{c=o}}$ compared to 1.44 and 3.55 for rGO respectively. This relation expresses the increased oxy-functionalization of rGO used in this study. The increase in the binding energy for the $\mathrm{sp}^{2}$ carbon peak from $\mathrm{rGO}(284.39 \pm 0.0598)$ compared to that of graphene $(284.13 \pm 0.154)$ results in an increase of $0.26 \mathrm{eV}$ which represents the oxyfunctionalization state of the carbon atoms. The shift in the peak is due to the increased electronegativity from the oxygen. The peak shifts of $\mathrm{C}-\mathrm{O}$ and $\mathrm{C}=\mathrm{O}$ are inside the error tolerance for the fits and are not comparable. 
S8. Optical characterization of the anode electrode

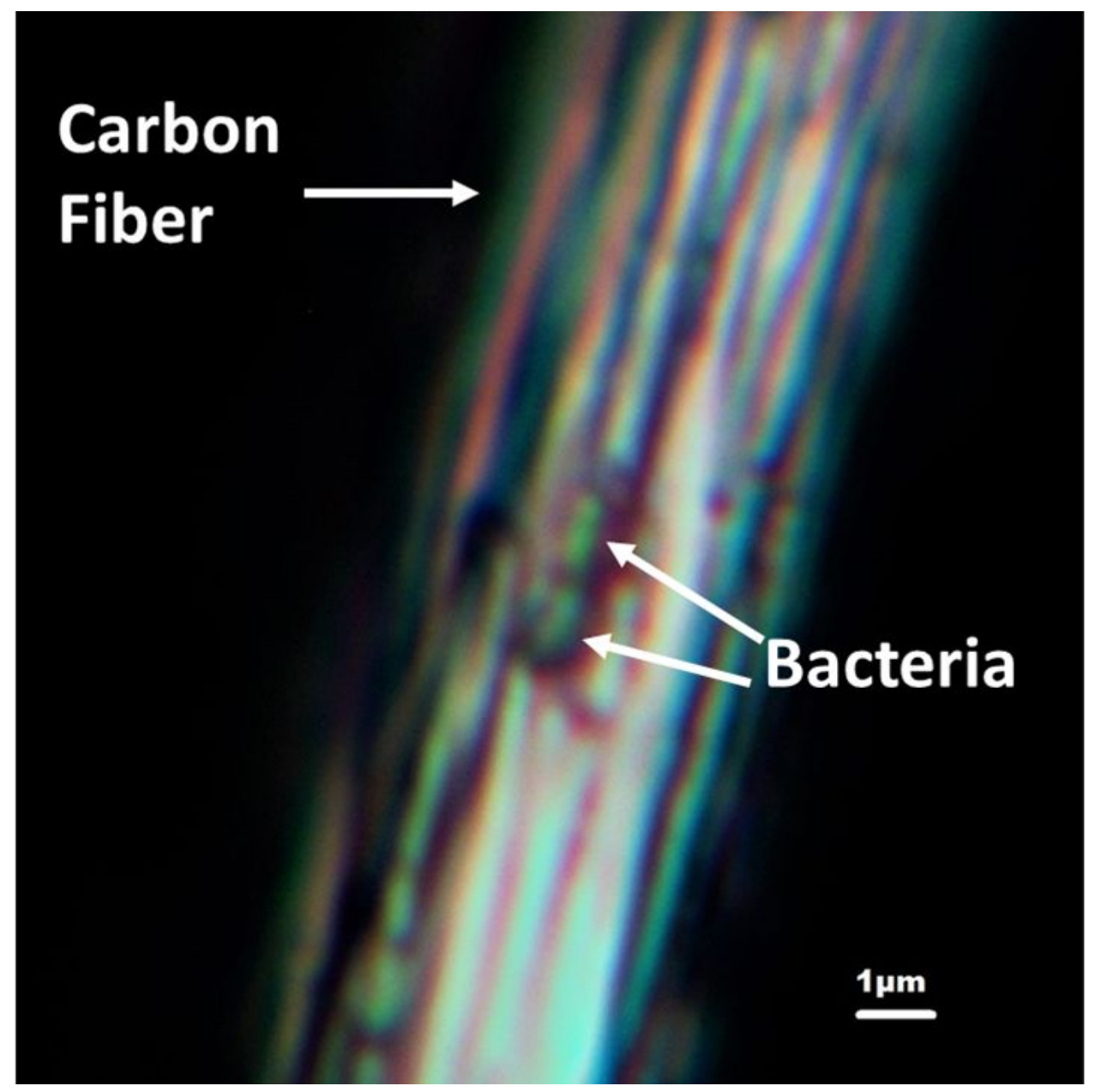

Figure S6. Optical image of a carbon fiber from an electrode after an experiment showing the adherence of two Geobacter bacterium to the surface. 
S9. Additional substrate effect on device performance

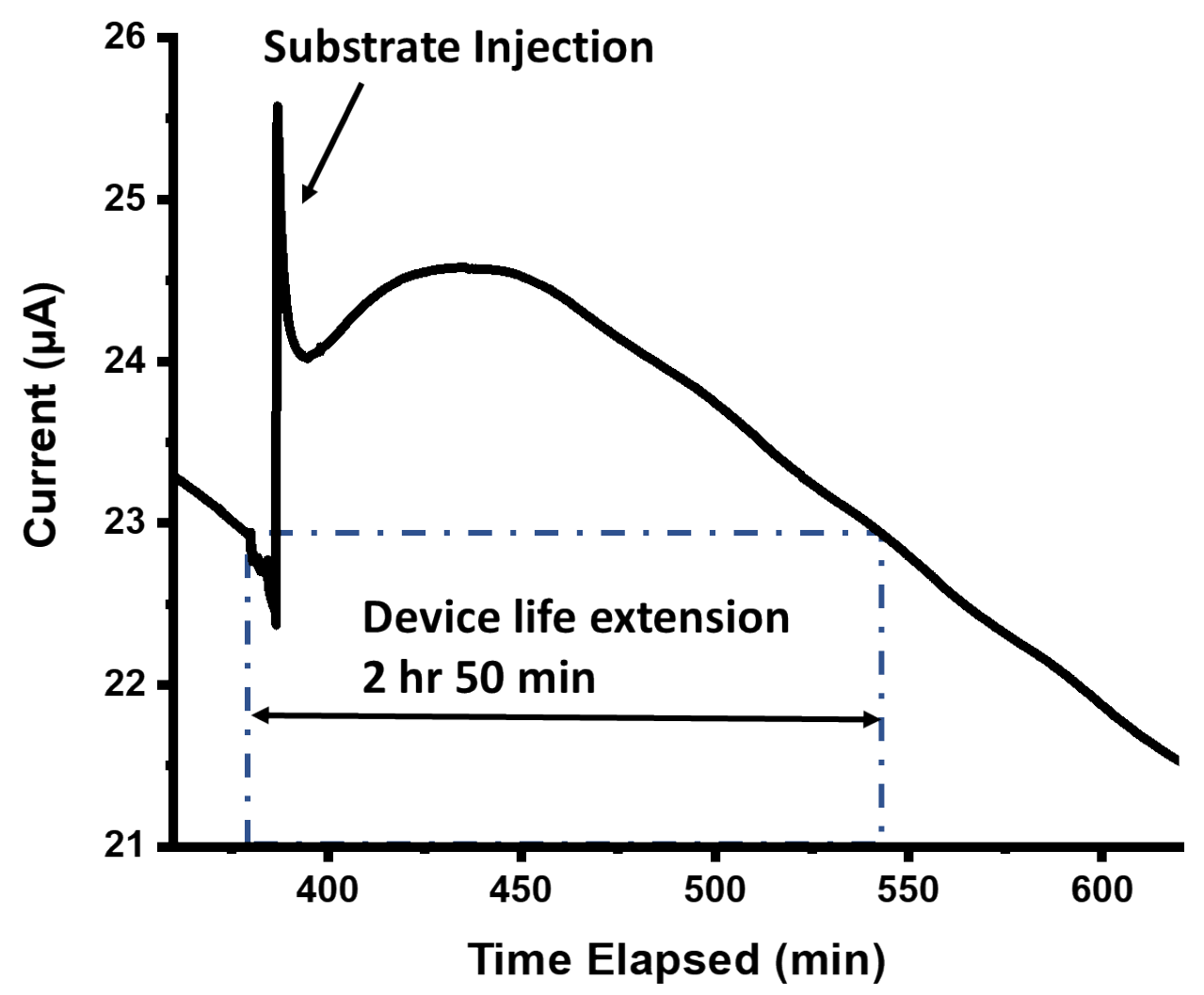

Figure S7. Current vs time chart showing the additional substrate injection. The device recovers current, as well as device lifetime due to the excess substrate $(0.2 \mathrm{M})$ in the system. An instant convection increase is observed upon the delivery of the substrate on the electrode surface inside of the anodic chamber. 